\title{
Alveolar Ridge
}

National Cancer Institute

\section{Source}

National Cancer Institute. Alveolar Ridge. NCI Thesaurus. Code C89749.

The thickened bony structures in the mandible and maxilla that contain the sockets of the teeth. 